\title{
HOT PHOTOLUMINESCENCE IN CdTe/CdMnTe QUANTUM WELL STRUCTURES GROWN BY MOLECULAR BEAM EPITAXY
}

\author{
M. Godlewski, T. WoJtowicz, G. Karczewski, J. Kossut \\ Institute of Physics, Polish Academy of Sciences \\ Al. Lotników 32/46, 02-668 Warsaw, Poland \\ J.P. Bergman, P.O. Holtz and B. Monemar \\ Department of Physics and Measurement Technology, Linköping University \\ 58183 Linköping, Sweden
}

A step-like emission is observed for CdTe/CdMnTe structures $\delta$-doped with In. The new photoluminescence cannot be explained by neither the Raman process nor by the "ordinary" hot photoluminescence. We propose that magnetic interactions are responsible for the new photoluminescence appearing due to a dramatic increase in a thermalization time of hot excitons.

PACS numbers: 78.47.+p, 78.55.Et

\section{Introduction}

Photoluminescence (PL) of CdTe/CdMnTe quantum well (QW) structures commonly consists of a two-line emission, which is related to a simultaneous observation of free (FE) and donor bound (DBE) excitons, observed even for undoped heterostructures [1]. In this work we report the observation of a new type PL, not observed for other QW structures studied by us. The origin of the new PL is discussed.

\section{Samples}

The CdTe/CdMnTe structures studied were grown by molecular beam epitaxy on (001) GaAs substrate covered with the $\mathrm{ZnTe} / \mathrm{CdZnTe}(10 / 20 \mathrm{~nm})$ superlattice and then with $3.5 \mu \mathrm{m}$ thick $\mathrm{CdTe}$ buffer layer. The active part of the structure consisted of 60 periods of $4 \mathrm{~nm}$ wide CdTe (well) $/ 13 \mathrm{~nm}$ wide CdMnTe (20\% Mn fraction, barrier). The first 10 periods were undoped. The following 50 periods were $\delta$-doped with indium to the level of $4 \times 10^{10} \mathrm{~cm}^{-2}$ either in the middle of the QW (sample \#1), or in the middle of the barrier (sample \#2) or both in the middle of the QW and the barrier (sample \#3). 


\section{Experimental results}

PL, PL excitation (PLE) and PL kinctics experiments were performed for all three structures studied. In Fig. 1 we show the PL spectrum measured at $2 \mathrm{~K}$ for the sample \#1 under two different resonant excitation conditions. The PLE spectra were measured to identify the origin of the PL bands. Based on the results of the PLE investigations we attribute a multiple double band structure of the PL to a simultaneous observation of free (FE) and donor bound (DBE) excitonic emissions form QWs of the nominal thickness and one monolayer (ML) larger $(\mathrm{ML}+1)$ and smaller (ML - 1). A larger width of the FE ML - 1 PL is due to an overlap of the $\mathrm{FE}$ emission, which is partly resolved at low excitation intensity, with a broad PL emission extending down in the energy. This emission is much broader than the FE and DBE bands and becomes stronger at higher excitation densities. Its origin is discussed below. The PL spectrum in Fig. 1 also shows an unidentified peak (labelled with ?) located in between the FE ML and the DBE ML emissions. We tentatively attribute this $\mathrm{PL}$ emission to the so-called $\mathrm{X}^{-}$transition (exciton consisting of two electrons and one hole) [2], which we expected for the modulation doped QW structures.

A simpler PL spectrum was observed for the sample \#2 with a two band structure related to a simultaneous observation of PL from QWs of the nominal thickness (ML) and from QWs one monolayer thinner ( $M L-1)$.

In addition to the complicated excitonic part of the PL we observed for all three heterostructures studied a step-like PL at the high energy wing of the PL. Detailed PL investigations were performed to determine the origin of the PL steps. Figure 2 shows that the PL steps appear at each multiple of the LO-phonon energy below the excitation energy. The step-like structure shifts with a shift of the excitation encrgy.

The temperature dependence of the PL intensity was measured. DBE PLs and the step-like PL are deactivated fast with the increasing temperature. They are not observed above $70 \mathrm{~K}$. FE PLs coming from the ML and ML \pm 1 QWs are

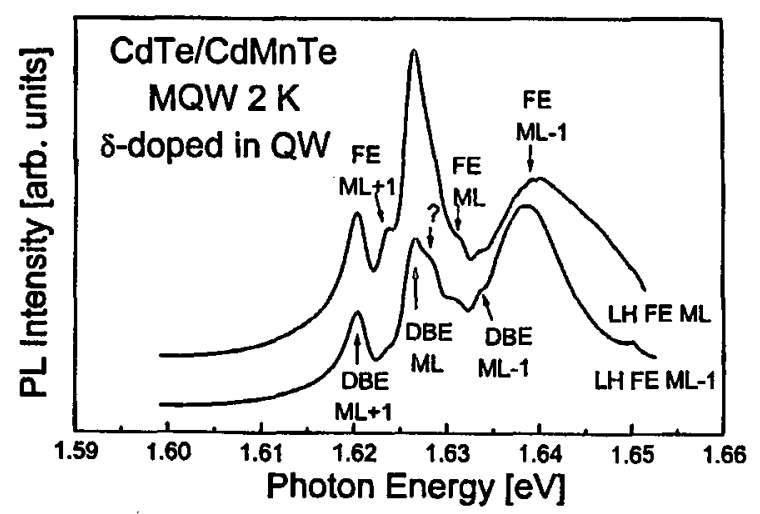

Fig. 1. The excitonic part of the photoluminescence spectrum of the sample \#1 measured at $2 \mathrm{~K}$ under two resonant excitation conditions. 


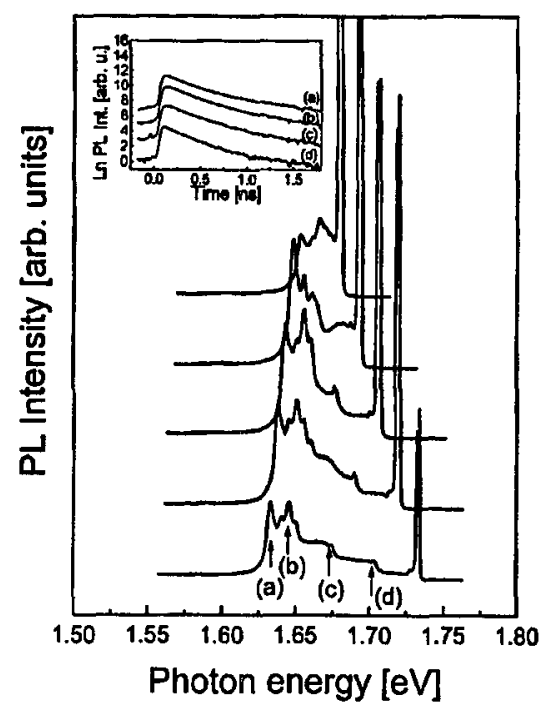

Fig. 2. Photoluminescence spectrum of the sample \#2 measured at $2 \mathrm{~K}$ under a pulsed excitation (sharp structure at high energy) set at different energies. In the inset we show PL kinetics measured at the detection set at different encrgies (labelled with letters) within the broad PL band.

still observed at $100 \mathrm{~K}$ and higher temperatures and the ratio of their intensity is not significantly changed by varying temperature of the experiment. This result indicates that these PLs are not coming from QW islands of a thickness varying by $1 \mathrm{ML}$ present in a one QW, but that they come from separated QWs. The process of the transfer is thus a phonon-assisted inter-QW exciton transfer proceeding through the CdMnTe barriers.

We performed $\mathrm{PL}$ kinetics investigations in picoseconds time range. In the inset of Fig. 2 we show the PL kinetics measured for the sample \#2 with the detection set at different energies (indicated with letters) within the PL spectrum. Identical PL kinetics is observed at different photon energies within the whole PL emission. Also a very similar PL kinetics is observed at LO-phonon peaks at the edge of the high energy wing of the PL.

\section{Discussion}

A hot PL was recently reported for $\mathrm{ZnTe} / \mathrm{MnTe}$ [3], ZnMnSe [4] and GaAs/AlGaAs [5]. In all these cases a series of LO-phonon peaks was observed below the laser line. Transient PL investigations showed for the ZnTe film and for the $5 \mathrm{LO}$ phonon replica a 10 ps decay time, which was longer from that of the laser line but was much shorter than that of the main PL spectrum [3]. A longer PL decay time means that those LO-phonon peaks are not due to the Raman process. It was proposed that LO-phonon replica are due to the phonon-assisted emission of separately localized electrons and holes. Thus, the LO-phonon replicas should appear in all the systems with localized carriers/excitons. 
The present experimental results cannot, however, be explained by neither the Raman effect nor by the hot PL process of the type discussed above. Instead of the separate LO-phonon replicas we observed PL steps starting at each multiple of the LO-phonon energy with only very weak additional peaks at LO phonon energy. To our knowledge such step-like PL was not observed for other systems. To explain its origin we must introduce the mechanism, which accounts for a slow relaxation of either hot electrons and holes or more likely hot excitons, occurring after an initial fast relaxation (a very fast rise time of the high energy wing of the PL is observed in the PL kinetics experiments). Exciton relaxation is slightly faster by the LO-phonon emission and is slightly slower by the acoustic phonon emission. The latter explains the appearance of the steps starting at each multiple of the LO-phonon energy with only weak additional peaks at exact multiples of the LO-phonon energy.

Even though the detailed origin of the interaction remains unknown, we relate the slow excitons thermalization to enhanced magnetic interactions in the $\mathrm{CdTe} / \mathrm{CdMnTe}$ structures studied rather than to the doping procedure. A step-like PL was not observed for other doped QW structures studied by us. We propose thus that the enhanced magnetic interactions are due to the strong inter-barrier coupling of magnetic moments of $\mathrm{Mn}$ ions in the CdMnTe barriers separated by thin CdTe QWs. We show separately that such coupling may result in several new phenomena such as e.g. an inter-QW exciton transfer [6].

Due to a strong exciton-Mn coupling, magnetic moments of $\mathrm{Mn}$ ions are polarized and magnetic polarons are formed. We have observed previously that this process typically occurs in a time scale of about $20-30 \mathrm{ps}$ (i.e., comparable to the PL rise time) or shorter and is faster than exciton thermalization time observed in the present study. The energy thermalization of inequilibrium (hot) magnetic polarons is much slower than that of free excitons and proceeds in a time scale longer than the typical PL decay time. This likely can be explained by strong spin selection rules of a coupled system of excitons and Mn ions.

This work was partly supported by the Committee for Scientific Research grants Nos. 8T 11B 01411 and 2P 03B 08709.

\section{References}

[1] M. Godlewski, M. Surma, G. Karczewski, J. Jaroszyński, T. Wojtowicz, J. Kossut, J.P. Bergman, B. Monemar, J. Cryst. Growth 159, 989 (1996).

[2] K. Kheng, R.T. Cox, Y. Merle d'Aubigné, F. Bassani, K. Saminadayar, S. Tatarenko, Phys. Rev. Lett. 71, 1752 (1993).

[3] N. Pelekanos, J. Ding, Q. Fu, A.V. Nurmikko, S.M. Durbin, M. Kobayashi, R.L. Gunshor, Phys. Rev. B 43, 9354 (1991).

[4] D. Some, A.V. Nurmikko, Phys. Rev. B 48, 4418 (1993).

[5] B.M. Ashkinadze, E. Tsidilkovski, E. Linder, E. Cohen, A. Ron, L.N. Pfeiffer, Phys. Rev. B 54, 8728 (1996).

[6] M. Godlewski, M. Surma, Z. Wilamowski, T. Wojtowicz, G. Karczewski, J. Kossut, P.O. Holtz, J.P. Bergman, B. Monemar, Acta Phys. Pol. A 92, 761 (1997). 